\title{
Effects of the Internal Shear Wave Window: Comparison With Anisotropy Induced Splitting
}

\author{
ENRU LIU ${ }^{1}$ and STUART CRAMPIN
}

British Geological Survey, Edinburgh, Scotland

\begin{abstract}
In the absence of anisotropy, the observed polarizations of shear waves should be those radiated from the source, modified only by interaction with internal interfaces and free surface topography. It has been suggested that shear wave polarizations may be systematically distorted by in ternal interfaces, which could lead to difficulties in interpreting anisotropyinduced shear wave splitting. Since the literature now reports many investigations of shear wave splitting, possible complications caused by internal interfaces would be important and need to be assessed. In this paper, we investigate the polarization of shear waves in full-wave synthetic seismograms incident from a point source at an isotropic-to-isotropic interface, over a range of angles of incidence. Particle motion due to plane and curved wave fronts has different characteristics. Internal shear wave windows can be defined, analogous to the shear wave window at the free surface, Within the innermost window, the polarizations at subsurface geophones (as in vertical seismic profiles) are recorded with little distortion, but outside this window the waveforms become increasingly distorted for larger angles of incidence. This is similar to the behavior of shear waves with respect to the shear wave window at the free surface, except that the behavior at internal interfaces is more complicated because of additional critical angles, and additional windows. We conclude that the interaction of shear waves with internal interfaces is only a serious difficulty when interpretating wide offset vertical seismic profiles, wide angle reflections, and cross-hole data.
\end{abstract}

\section{INTRODUCTION}

In this paper, we examine the effects on shear waves of transmission through in ternal interfaces, which leads to the concept of a series of internal shear wave windows analogous to the shear wave window previously defined for incidence at a free surface [Evans, 1984]. The scattering of shear wave polarizations at the free surface has been discussed by Nutlli [1961, 1964], Nutlli and Whitmore [1962], Evans [1984], and Booth and Cramp in [1985]. They showed that shear waves observed at a free surface may be seriously distorted by interaction with a free surface if the angle of incidence is greater than the critical angle arcsin $\left(V_{s} / V_{p}\right)$. This angle defines a shear wave window, within which the shear waveforms observed at the surface are similar to the waveforms of the incident waves [Booth and Crampin, 1985]. The particle motions of shear waves arriving outside the window are severely distorted and the waveforms of the incident wave cannot easily be recovered from observations at the free surface.

Recent studies have shown that interaction with internal interfaces may also distort shear wave polarizations. Cormier [1984] has concluded that

\footnotetext{
${ }^{1}$ Also a t Department of Geology and Geophysics, University of Edinburgh, Scotland.
}

Copyright 1990 by the American Geophysical Union.

Paper number $90 \mathrm{JB} 80264$.

0148-0227/90/90JB-00264\$05.00 transmission through irregular internal interfaces could lead to distortions of shear wave polarizations of up to $10^{\circ}$. Douma and Helbig [1987] calculated the change in polarization of a plane shear wave polarized intermediate to $S V$ and SH transmitted through an interface between halfspaces of sandstone and halite at a range of angles of incidence. They find that the deviation is greatest $\left(3^{\circ}\right)$ at an angle of incidence of about $30^{\circ}$.

Douma and Heibig [1987] suggest that such effects are cumulative and might have serious implications for the study of anisotropy-induced shear wave splitting. Shear wave splitting is caused by internal structure, usually by some form of effective anisotropy, but internal interfaces can also cause differences in the response of the polarizations of shear waves that might be mistaken for anisotropy-induced splitting. Anisotropy-induced shear wave splitting in the Earth's crust appears to be caused by distributions of stress-aligned fluid-filled cracks, microcracks, and preferentially oriented pore-space, known as extensive dilatancy anisotropy or EDA [Crampin e I al., 1984; Crampin. $1985 \mathrm{~b} ; \quad$ Crampinand Atkinson, 1985]. The inclusions themselves are known as EDA cracks since many of the seismic effects can be simulated by propagation through parallel vertical microcracks, al though it is recognized that the inclusions may take a wide range of shapes from cracks and microcracks, to preferentially oriented pore space. Splitting due to such EDA cracks is now observed along almost all ray paths in the uppermost 10 to $20 \mathrm{~km}$ of the crust [ Crampin, 1987], and is currently exciting 
much interest both in earthquake and exploration geophysics.

If internal interfaces cause difficulties in interpreting anisotropy-induced shear wave splitting, as sugges ted, the effects need to be quantified. In this paper, we calculate synthetic seismograms of shear waves incident on internal isotropic-to-isotropic and isotropic-toanisotropic interfaces, and assess the effects of such interfaces on observations of anisotropy induced shear wave splitting. The synthetic seismograms are calculated with the modified reflectivity technique of ANISEIS (previously called ROCPAC, [Taylor, 19851). An internal shear wave window is defined, within which the polarizations of transmitted shear waves are recorded with little distortion. This paper on the internal shear wave window may be considered as a continuation of and addendum to the paper by Booth and Crampin [1985] on the shear wave window at the free surface.

\section{BEHAVIOR OF POLARIZATIONS OF PLANE WAVES AT AN ISOTROPIC-TO-ISOTROPIC INTERFACE:} ANALYTICAL SOLUTIONS

Analytical solutions are only available for plane waves incident on plane interfaces. We consider two examples. Incidence at a low-tohigh impedance contrast, L/H, (the case considered by Douma and Helbig [1987]), and incidence at a high-to-low impedance contrast, H/L. This classic and fundamental problem has been treated in many investigations [for example, Ewing et al. , 1957; Brekhovsk $i \mathrm{kh}, 1960 ;$ Aki and Richards, 1980]. In this section, we shall consider a plane wave impinging upon a plane welded boundary.

\subsection{Polarization Angles}

We consider a plane shear wave with relative $S H$ and $S V$ amplitudes $A_{S H}$ and $A_{S V}$ respectively, incident on a plane boundary from Material 1 to Material 2 with parameters in Table 1 . The polarization angle or vibration angle, $\psi$, of the transmitted shear wave in the plane parallel to the plane of constant phase is related to the amplitude and transmission cofficients [ Ingram. 1952 ; Douma and Helbig, 1987] by

$$
\psi=\tan ^{-1}(B|R|)
$$

where $B={ }_{S H} / A_{S V} ;$ and $R=T_{S H} / T_{S V}$ The ratio $B=A_{S H} / A_{S V}$ specifies the polarization

TABLE 1. Velocities and Densities of Sandstone and Halite

\begin{tabular}{lccl}
\hline & $\begin{array}{c}V_{P}, \\
\mathrm{~km} / \mathrm{s}\end{array}$ & $\begin{array}{c}V_{S^{\prime}} \\
\mathrm{km} / \mathrm{s}\end{array}$ & $\begin{array}{c}\rho, \\
\mathrm{g} / \mathrm{cm}^{-3}\end{array}$ \\
\hline $\begin{array}{l}\text { Material 1 } \\
\text { (sandstone) }\end{array}$ & 3.074 & 1.904 & 2.65 \\
$\begin{array}{l}\text { Material 2 } \\
\text { (halite) }\end{array}$ & 4.618 & 2.697 & 2.16 \\
\hline
\end{tabular}

From Douma and Helbig [1987]. angle of the incident wave, and $T_{S H}$, and $T_{S V}$ represent the transmission coefficients of $S H$, and $S V$ waves, respectively, which are functions of of properties of the materials $\left(\rho_{1}, V_{S l}, V_{P l}\right.$ and $\left.\rho_{2}, \quad V_{S 2}, V_{P 2}\right)$ and angle of incidence $i[A k i$ and Richards, 1980]. Figure 1 defines the shear wave polarization angle, $\psi$.

Figure 2 shows the polarization angle and phase difference of the transmitted shear wave, for an incident wave with equal amplitudes of $S H$ and $S V$ waves $\left(\psi=45^{\circ}\right.$, and $B=1$, which we shall call SH45SV, as functions of the angles of incidence for the two different interfaces. Figure $2 a$

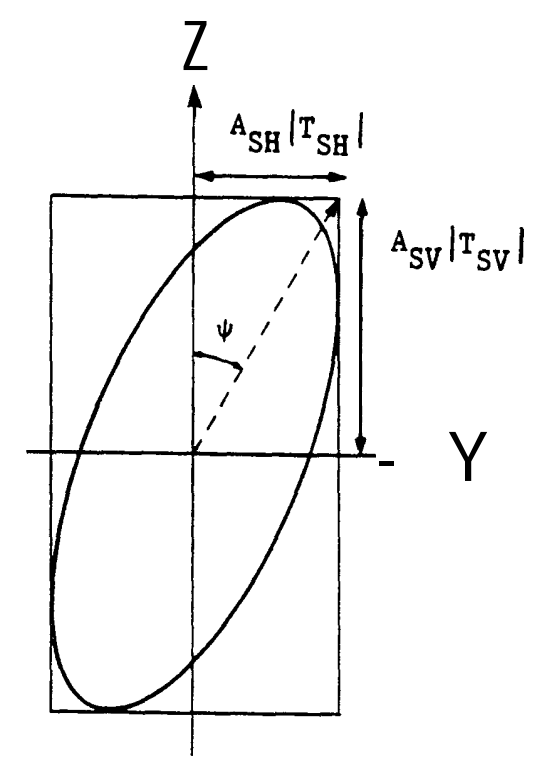

Fig. 1. Definition of polarization angle $\psi$ of a shear wave in the plane parallel to the plane of constant phase. $A_{S H}$ and $A_{S H}$ are the amplitudes of the $S H$ and $S V$ componentso the incident shear wave. $T_{S H}$ and $T_{S V}$ are the transmission coefficients $Q S_{S} H_{S H}$ and $S V V$ waves.

shows polarization angle and Figure $2 b$ the phase difference for sandstone to halite, a low-to high-velocity $(\mathrm{L} / \mathrm{H})$ interface; and Figures $2 c$ and $2 d$ similar values for halite to sandstone, a high- to low-velocity $(\mathrm{H} / \mathrm{L})$ interface. Figure $2 a$ is a recalculation of Figure 9 in the paper by Do uma and Helbig [1987], but calculated for a full range of angles of incidence from $0^{\circ}$ to $90^{\circ}$. We see that at normal incidence $\left(i=0^{\circ}\right)$, the polarization and phase- of the transmitted wave are unchanged. The deviation of the polarization and phase of the transmitted wave increases as the angle of incidence increases, reaching $3^{\circ}$ for $\mathrm{L} / \mathrm{H}$, and $2^{\circ}$ for $\mathrm{H} / \mathrm{L}$ at the smallest critical angle, $\arcsin \left(V_{S l} / V_{P 2}\right)$ for $\mathrm{L} / \mathrm{H}, \quad$ and $\arcsin$ $\left(V_{S 2} / V_{P 2}\right)$ for $\mathrm{H} / \mathrm{L}$, respectively. Beyond these critical angles, the incident wave is totally reflected, and there is an inhomogeneous interface wave, with energy propagating parallel to, and decaying exponentially away from, the interface.

\subsection{In terface Waves}

When a linearly polarized plane shear wave is transmitted through an isolated isotropic-to- 

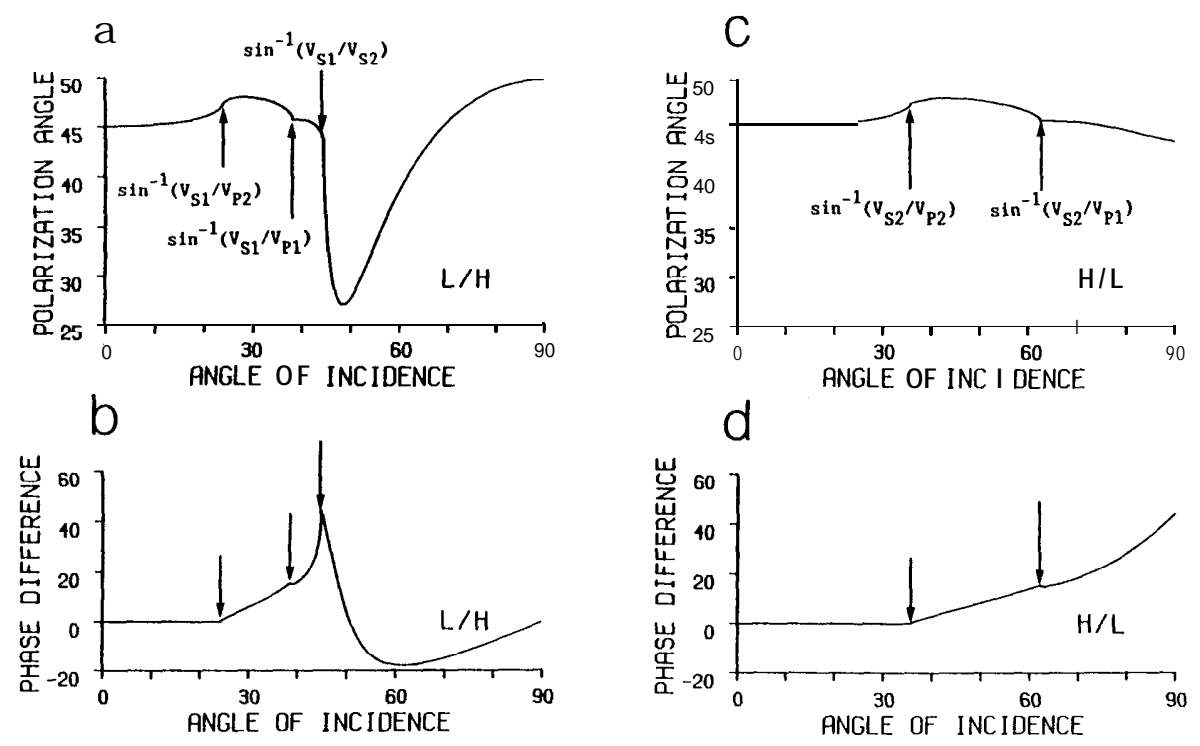

Fig. 2. ( $a$ and c) Polarization angles $\psi$ of transmitted shear waves, and ( $b$ and $d$ ) phase differences, as functions of incident angles $i$ for ( $a$ and $b$ ) sandstone-to-halite interface, $\mathrm{L} / \mathrm{H}$, and (c and $d$ ) halite-to-sandstone interface, H/L. The ray paths at critical angles of incidence are marked with arrows.

isotropic interface, within the innermost window (incidence less than the smallest critical angle), the phase and the (linear) motion of the incident wave are preserved. The particle motion becomes elliptical only for the angles of incidence greater than the smallest critical angle. There are usually three critical angles for $\mathrm{L} / \mathrm{H}$ $\left(V_{P 1}<V_{P 2}, V_{S l}<V_{S 2}\right)$ defined, in order of increasing angle, by

$$
\begin{aligned}
& { }_{C l}=\sin ^{-1}\left(v_{S l} / v_{P 2}\right) \\
& { }_{C 2}=\sin ^{-1}\left(v_{S l} / V_{P l}\right) \\
& { }_{C 3}=\sin ^{-1}\left(v_{S l} / v_{S 2}\right)
\end{aligned}
$$

and two critical angles for $\mathrm{H} / \mathrm{L}\left(V_{P I}>V_{P 2}, V_{S I}>\right.$ $v_{S 2}, \quad v_{\mathrm{P} 1}>v_{\mathrm{S} 2}$ ) defined by

$$
\begin{aligned}
& { }_{\alpha_{C 4}}=\sin ^{-1}\left(v_{S 2} / v_{P 2}\right) \\
& { }_{C 5}=\sin ^{-1}\left(v_{S 2} / v_{P 1}\right)
\end{aligned}
$$

As the angle of incidence exceeds the smallest critical angle, the coefficients of the previously real transmitted wave become complex, and the resultant inhomogenous interface waves become elliptically polarized. The polarization ellipse in the plane containing $S H$ and $S V$ components was described by Smi th and Ward [1974] and Bullen and Bol I [1985], and is determined by the following equation

$$
\begin{gathered}
\sin ^{2}(\Delta \phi)=y^{2} /\left(A_{S H}\left|T_{S H}\right|\right)^{2}+z^{2} /\left(A_{S V}\left|T_{S V}\right|\right)^{2} \\
-2 y z \cos (\Delta \phi) /\left(A_{S H} S_{S V}\left|T_{S H} T_{S V}\right|\right)
\end{gathered}
$$

where the phase difference is $\mathrm{A}+=\phi_{S H}-\phi_{S V}$ and $\phi_{S H}$ and $\phi_{S V}$ are the phase angles of $S H$ and $S V$ waves, respectively. The elliptical polariza- tions are inhomogeneous waves carrying energy parallel to the interface at the phase velocity of the incident shear wave along the interface [ Hudson, 1980; Kenne t $t, 1983$ ].

The polarization angles show abrupt changes at the three critical angles for $\mathrm{L} / \mathrm{H}$ (Figure 3 a), $24.34^{\circ}, 38.27^{\circ}$, and $44.91^{\circ}$, corresponding to the limits of transmitted Pwaves, reflected Pwaves, and transmitted shear waves, respectively, and two critical angles for H/L (Figure 2c), 35.73' and $61.32^{\circ}$, corresponding to the limits of reflected $P$, and refracted Pwaves, respectively. The deviations of the polarizations for the inhomogeneous wave near the interface in $\mathrm{L} / \mathrm{H}$ may be up to $20^{\circ}$ with a phase difference of also about $20^{\circ}$. It follows therefore, that the critical angle ${ }^{\alpha}{ }_{C 3}=\arcsin \left(V_{S 1} / V_{S 2}\right)$ is important for $\mathrm{L} / \mathrm{H}$, and may indicate the beginning of strongly elliptical motion. The inhomogeneous waves are less elliptical for $\mathrm{H} / \mathrm{L}$ as the largest deviation is only $4^{\circ}$, which in many circumstances may be negligible.

\section{POLARIZATIONS OF SHEAR WAVES WITH CURVED WAVE FRONTS AT ISOTROPIC-TO-ISOTROPIC} INTERFACES

The variations of polarization diagrams (PDs) of a $20 \mathrm{~Hz}$ pulse $\exp (-2 \pi 20 \mathrm{t}) \sin (2 \pi 20 \mathrm{t})$, shown in Figure $3 a$, of $S H 45 S \mathrm{~V}$ waves radiated from a point source $1 \mathrm{~km}$ above $\mathrm{L} / \mathrm{H}$ and $\mathrm{H} / \mathrm{L}$ interfaces have been calculated for an equispaced grid of geophones with the simple geometry of Figure $3 \mathrm{~b}$. R, $\mathrm{T}$ and $\mathrm{V}$ indicate the horizontal radial, transverse and vertical directions, respectively. The lines in Figure 3 mark the ray paths at critical angles of incidence for the $\mathrm{L} / \mathrm{H}$ interface. The PDs for $\mathrm{L} / \mathrm{H}$ and $\mathrm{H} / \mathrm{L}$ are presented in Figure 4. The particle motions show true relative amplitudes for a fixed amplitude incident wave in three mutually perpendicular PDs in vertical/ 
a

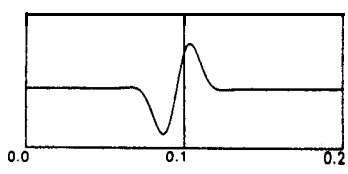

b

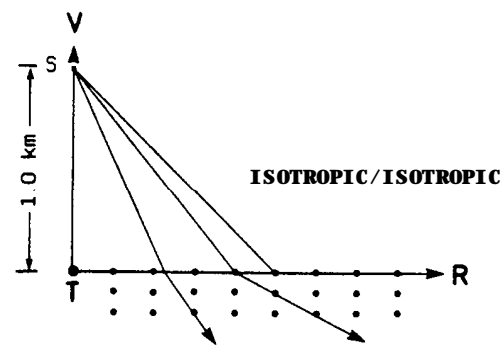

Fig. 3. (a) $20-\mathrm{Hz}$ source pulse used for polarization diagrams. (b) Schematic illustration of the model for shear wave propagation across a plane isotropic-to-isotropic interface at a range of angles of incidence. A $20-\mathrm{Hz}$ point source is located $1 \mathrm{~km}$ above the interface. The transmitted shear waves are recorded at depths of $0.001,0.1$, and $0.2 \mathrm{~km}$ below the interface. Three critical angles defined by $a_{c}=\arcsin$ $\left(v_{S 1} / v_{P 2}\right), \alpha_{C 2}=\arcsin \left(v_{S 1} / v_{P 1}\right)$, and $\alpha_{C 3}=$ $\arcsin \left(V_{S I} / V_{S 2}\right)$ for $\mathrm{L} / \mathrm{H}$ are indicated. Vertical (V), horizontal radila (R), and transverse (T) directions are marked. The wavelengths of the shear waves are $95 \mathrm{~m}$ in the sandstone and 135 $\mathrm{m}$ in the halite.

radial, vertical/transverse, and horizontal planes. The patterns in the PDs uniformly decrease to very small sizes for wider angles of incidence and have been omitted for economy of space.

The ray paths at critical angles of incidence are marked in Figure 4 . The critical ray paths mark successive increases of ellipticity of the polarization diagrams for the near-interface recording points. At greater distance from the interface, the polarization diagrams only show minor differences on either side of the ray path of critical incidence. The vertical-transverse plane for the $\mathrm{L} / \mathrm{H}$ interface in Figure $4 a$ shows most ellipticity, but in all cases the ellipticity is comparatively weak except at large angles of incidence. The results shown here are in general consistent with the theoretical behavior of plane waves at an internal interface in Figure 2. The small differences are caused by the different behavior of plane and curved wave fronts at interfaces.

The presence of several critical angles of incidence at a plane internal interface makes the behavior of shear waves at the interface more complicated, but the effects are less severe than the behavior at a free surface. At the free surface, a shear wave arriving outside the shear wave window is severely distorted [Booth and Crampin, 1985 ]. At an internal interface, except for large angles of incidence, the principal effects of the interface are confined to the successive elimination of transmitted waves and excitation of interface waves beyond the critical angles.
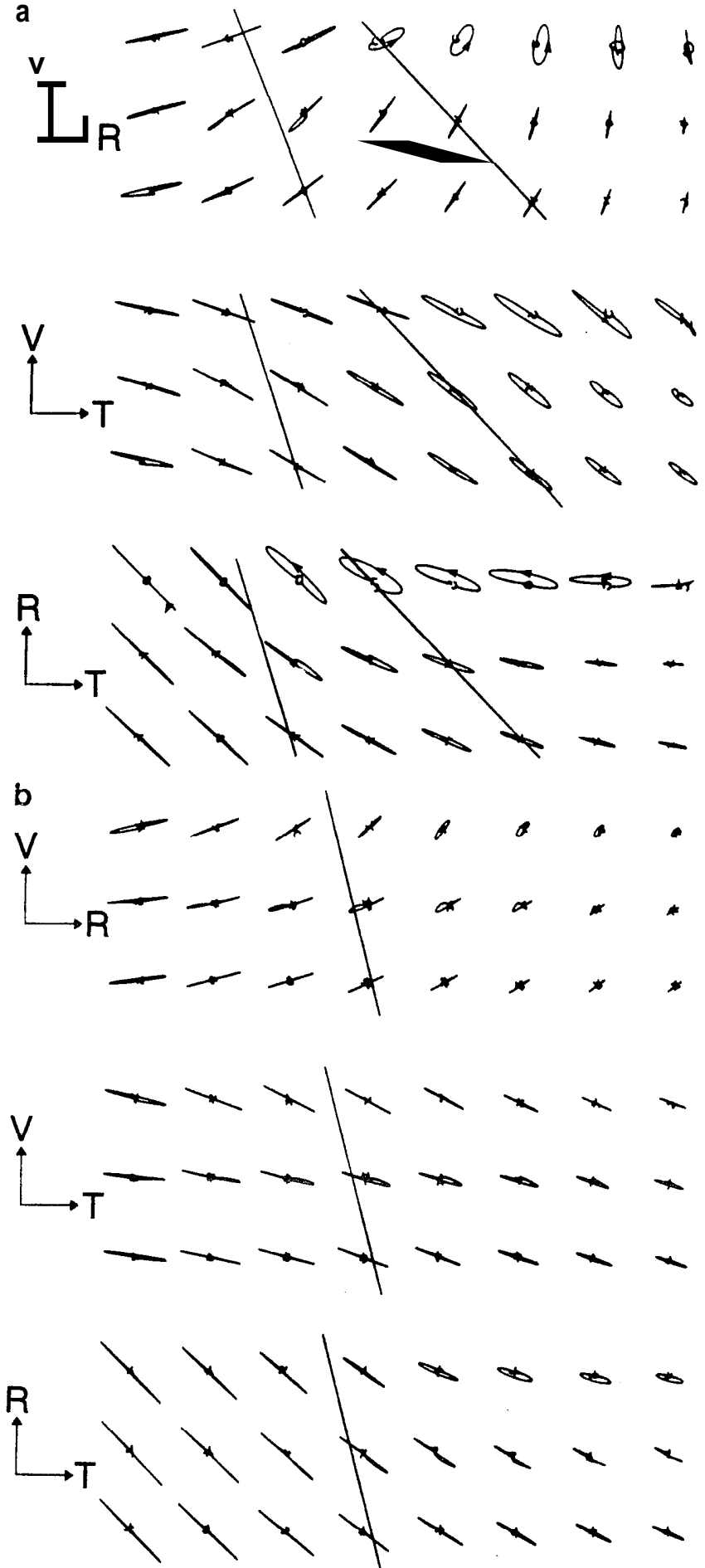

Fig. 4. Polarization diagrams for three mutually perpendicular sections V-R, V-T, and R-T, for shear waves transmitted through an interface from a point source with the geometry in Figure 3 . Notation as in Figure 3. (a) Sandstone-to-halite $(\mathrm{L} / \mathrm{H})$ interface. (b) Halite-to-sandstone $(\mathrm{H} / \mathrm{L})$ interface. The incident pulse is a 20-Hz SH45SV wave. The ray paths at the critical angles of incidence are shown. Small arrowheads mark the direction of motion. .

We may define internal shear wave windows in the same way as at the free surface, within which the shear waves are recorded with little distortion at the interface. Similar to the behavior 
a
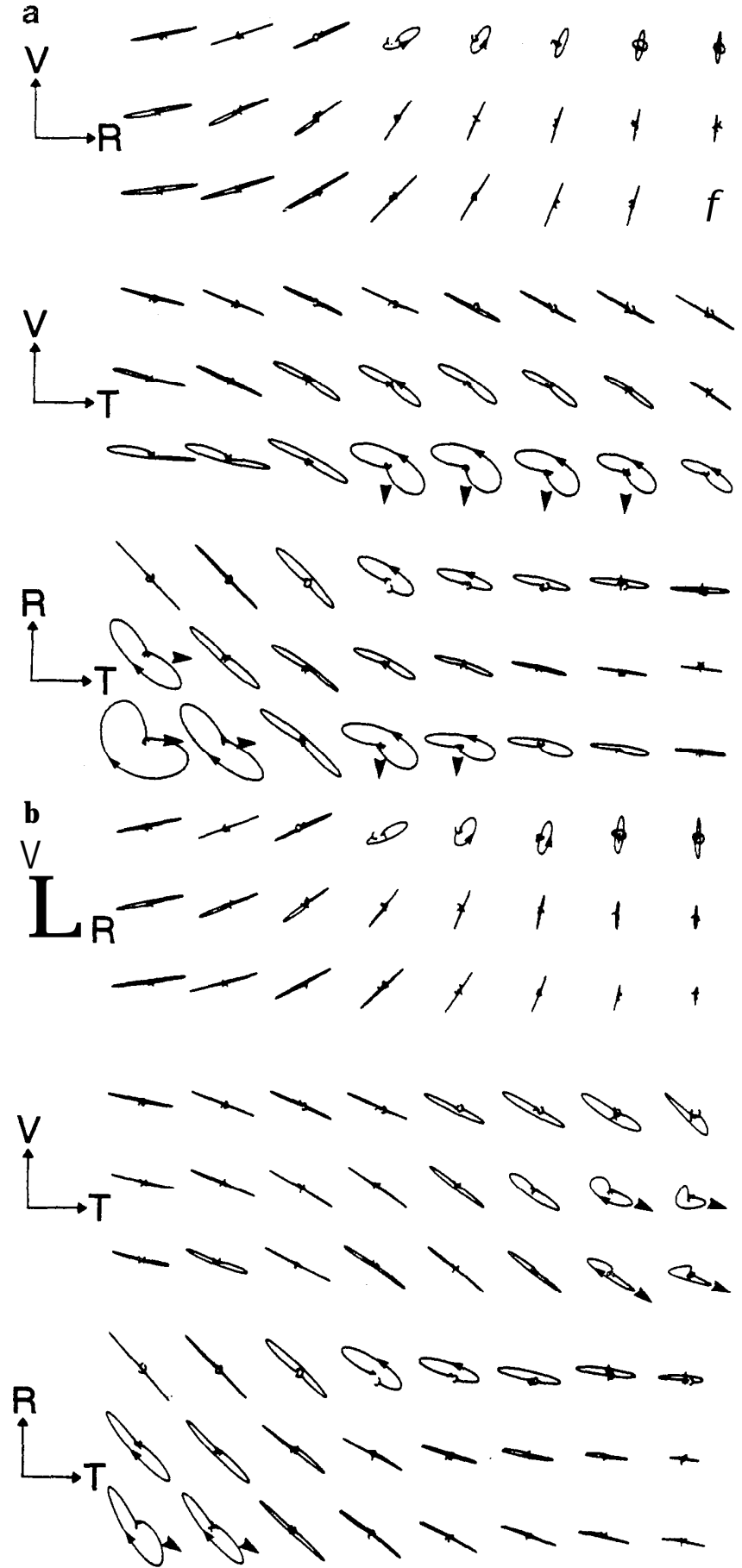

Fig. 5. Transmission through an isotropic-toanisotropic interface, with the notation of Figure 4. The isotropic material is sandstone (Table 1), and the anisotropy is a distribution of parallel vertical thin fluid-filled cracks in a halite matrix with elastic constants in Table 2. The takeoff directions of initial shear wave motion are marked with large arrowheads and the direction of motion with small arrowheads.

(a) cracks striking perpendicular to the source/ geophone spread, and (b) cracks striking $30^{\circ}$ clockwise from transverse direction.

at the shear wave window at the free surface [Bootha $n d$ Crampin, 1985], critical angles are exactly defined and mark abrupt changes of particle motions only for plane waves. On curved wave fronts as in Figure 4, the effects of the critical angle are spread over a range of angles, the exact behavior for any particular geometry depending on the curvature of the wave front and the frequency of the incident wave.

\section{COMPARISON OF THE EFFECTS OF INTERFACES AND ANISOTROPY-INDUCED SHEAR WAVE SPLITTING}

\subsection{Isotropic-to-Anisotropic Interface}

We calculate the behavior of shear waves at an isotropic-to-anisotropic interface, where the anisotropy is caused by distributions of vertical parallel fluid-filled microcracks (EDA cracks, with elastic constants listed in Table 2). Figure 5 shows polarization diagrams of shear waves below an isotropic-to-anisotropic interface with the same geometry as the $\mathrm{L} / \mathrm{H}$ interface in Figure $4 a$ with the cracks striking $0^{\circ}$ (Figure $5 a$ ), and $30^{\circ}$ (Figure $5 b$ ), respectively, clockwise from the transverse direction, relative to the source-togeophone line. The velocities of the uncracked matrix are the same as halite in Figure $4 a$ so that the behavior of shear waves at isotropic-tois0 tropic and isotropic-to-anisotropic interfaces may be compared directly. The effects of the anisotropy only begin to be visible when the path length through the anisotropy is long enough for shear wave splitting to separate the two waves. Consequently, the polarizations of shear waves immediately below the interface are very similar to those for the isotropic-to-isotropic interface in Figure $4 a$, and a change from linear to elliptical motion occurs at the critical angle of incidence.

At receivers farther from the interface, the shear waves propagate for greater distances through the effective anisotropy and the effects of the anisotropy-induced shear wave splitting become dominant in the polarization diagrams, particularly in the horizontal and transverse planes. The shear wave polarization diagrams in the horizontal plane show strong ellipticity (pronounced splitting) at small angles of incidence, where in the isotropic model in Figure $4 a$, the shear waves are linearly polarized except at large angles of incidence. The first motions marked with arrowheads are parallel to the strikes of the aligned cracks at small angles of incidence.

In Figure 5a, and less noticeably in Figure 56, there is a sudden change in shear wave PD in the horizontal (radial-transverse) plane for the deeper geophones, where the rotation direction of particle motions changes from clockwise to linear, and then to anticlockwise rotation. This phenomenon is caused by the behavior of shear waves passing through the cracked medium. The change in the direction of rotation is caused by the intersection of the velocity curves of the two split shear wave polarizations at approximately $60^{\circ}$ from the crack normal $\left(30^{\circ}\right.$ from vertical) as has been shown in many publications, Crampin [1985a], for example. The polarization of the faster split shear wave changes by approximately $90^{\circ}$ on crossing this intersection.

\subsection{Shear Wave Split ring}

The shear wave splitting is a diagnostic characteristic of shear wave propagation through 
TABLE 2. Elastic Constants of the Anisotropic Material Used in Calculating the Synthetic Seismograms of Figure 5, Where the Velocities of the Uncracked Matrix Are the Same as Halite in Table 1

\begin{tabular}{ll}
\hline Constant & Value \\
\hline$c_{1111}$ & 45.87 \\
$c_{2222}=c_{3333}$ & 46.04 \\
$c_{1122}=c_{1133}$ & 14.58 \\
$c_{2233}$ & 14.62 \\
$c_{3131}=c_{1212}$ & 12.51 \\
\hline
\end{tabular}

Units are $\mathrm{Pa} \times 10^{9}$.

cracked and anisotropic solids. The possible orientations of the split shear waves are determined by the path through the anisotropic symmetry of the medium in which the geophone is situated, not the polarization of the incident shear wave. Thus, any disturbance to the shear wave polarizations caused by the interface, may change the relative proportions of the split shear waves into which the initial pulse splits, but will not alter the polarizations of the split components.

The shear wave splitting in Figure 5 shows several differences from the elliptical polarizations associated with interfaces shown in Figure 4. Within the shear wave window (incidence angle less than arcsin $\left(V_{S I} / V_{P 2}\right)$ for $\mathrm{L} / \mathrm{H}$ and arcsin $\left(V_{S 2} / V_{P 2}\right)$ for $\left.\mathrm{H} / \mathrm{L}\right)$, distinct shear

wave splitting may be seen. In particular, the polarization of the leading (faster) split shear wave is controlled by the orientation of the anisotropy (the strike of the EDA cracks) not by the polarization of the incident wave. This demonstrates that the small distortions in polarization introduced by the interface may modify the details of the pattern in the PDs, but generally will not alter the polarization direction of the leading split shear wave.

\section{CONCLUSIONS}

There are several concentric shear wave windows associated with the critical angles of incidence. Within the innermost window, the transmission coefficients of the shear waves are real, there are no phase changes, and the polarization of the incident shear wave in an isotropic structure is essentially preserved with only minor deviations of direction. Beyond this first critical angle, inhomogeneous interface waves excite motion with elliptical motion largely confined to the immediate neighborhood of the interface.

The effects of anisotropy are different from the interface effects and they cannot be confused. Passage through the inner shear wave window into an anisotropic layer produces shear wave splitting, with the delay between the split shear waves progressively increasing with the length of the anisotropic ray path. This gives typical polarization diagrams of shear wave splitting with orthogonal changes of direction which cannot be mistaken for interface-induced elongated ellipticity. At wider angles of incidence, the behavior of shear waves at isotropic-to-isotropic and isotropic-to-anisotropic interfaces are similar to each other only near the interface, when there has been insufficient anisotropic path length to cause significant shear wave splitting so that the effect of the interface is dominant. The interpretation of the splitting could only be mistaken if the delay between the split shear waves is very small, as a consequence of a short anisotropic ray path, or of weak anisotropy.

The important result is that the initial polarization of the shear waves in PDs is controlled by the anisotropy (the orientation of EDA cracks). The small changes in orientation of the wave caused by the interface will only affect details of the PD pattern not the initial polarization, thus visual identification of polarization directions in PDs will not be affected, and if synthetic seismograms are used for interpretation, the effects of the interface will be modeled in any case. We conclude that the interaction of shear waves with internal interfaces is unlikely to be a serious complication in observa$t$ ions and interpretations of anisotropy-induced shear wave splitting, although it may contribute to the usually observed complexity of the waveforms following the initial onset of the faster split shear wave.

Acknowledgments. We thank Applied Geophysical Software Inc. and Macroc Ltd. for approval to use the ANISEIS package for calculating the synthetic seismograms. We thank Michael Schoenberg for his review pointing out some errors in our understanding of interface waves. We also thank Iain Bush for valuable discussions. The sponsorship of one of the authors (E.L.) by the British Council and the Chinese Government is gratefully acknowledged. This work was supported by the Natural Environment Research Council and is published with the approval of the Director of the British Geological Survey (NERC).

\section{REFERENCES}

Aki, K., and P. G. Richards, Quantitative Seismology, Theory and Methods. vol. 1, W. H. Freeman, New York, 1980.

Booth, D. C., and S. Crampin, Shear-wave polarization on a curved wave front at an isotropic free surface, Geophys. J. R. Astron. Soc., 83, 31-45, 1985 .

Brekhovskikh, L. M., Waves in Layered Media, Academic Press, Sa n Diego, California, 1960.

Bullen, K. E., and B. A. Bolt, An Introduction to the Theory of Seismology, Cambridge University Press, New York, 1985.

Cormier, V. F., The polarizations of $s$ waves in a heterogeneous isotropic Earth's model, J. Geophys. Res., 56. 20-23, 1984.

Crampin, S., Evaluation of anisotropy by shear-wave splitting, G eophysics, 50, 142-152, 1985a.

Crampin, S., Evidence for aligned cracks in the Earth's crust, First Break, 3, 12-15, $1985 \mathrm{~b}$

Crampin, S., Geological and industrial implications of extensive-dilatancy anisotropy, Nature, 328, 491-496, 1987 .

Crampin, s., a n d B. K. Atkinson, Microcracks in the Earth's crust, First Break, 3, 16.20, 1985.

Crampin, S., J. R. Evans, and B. K. Atkinson, Earthquake prediction: a new physical basis, Geophys. J. R. As t ron. Soc. , 76, 147.156, 1984 .

Douma, J., and K. Helbig, What can the polarization of shear-waves tell us?, First Break, 5. 95-104, 1987. 
Evans, J. R., Effects of the free surface on shear waves, Geophys. J. R. As t ron. Soc. , 76. 165-172, 1984.

Ewing, W. M. , W. S. Jardetzky, and F. Press, Elastic Waves in Layered Media, McGraw-Hill, New York, 1957.

Hudson, J. A., The Excitation and Propagation of Elastic Waves, Cambridge University Press, New York, 1980.

Ingram, R. E., Vibration angles of S waves, Bull. Seismol. Soc. Am., 42. 145-151, 1952.

Kennett, B. N. K., Seismic Wave Propagation in Stratified Media, Cambridge University Press, New York, 1983.

Nuttli, O., The effect of the Earth's surface on the S-wave particle motion, Bull. Seismol. Soc. Am., $5 l$. $246,1961$.

Nuttli, O., The determination of $S$-wave polarization angles for an Earth model with crustal layering, Bull. Seismol. Soc. A m. , 54, 1429-1440, 1964.
Nuttli, O., and J. D. Whitmore, On the determination of the polarization of the S-wave, Bull. Seismol. Soc. Am. 52, 95-107, 1962.

Smith, B. D., and S. H. Ward, Short Note: On the computation of polarization ellipse parameters, Geophysics. 39 $867-869,1974$

Taylor, D. B., ROCPAC Manual: Sumary, Macroc, Edinburgh, 1985

S. Crampin and E. Liu, British Geological Survey, Murchison House, West Mains Road, Edinburgh EH9 3LA, Scotland, UK.

(Received June 22, 1989;

revised January 18, 1990; accepted December 17, 1989.) 